\title{
Dialéctica Irresuelta de la Arquitectura en el Escenario Digital
}

\author{
Indecisive Dialectics of the Architecture in the Digital Stage
}

\author{
Dayana T. Pirela Rivas \\ UNERMB - LUZ, Venezuela \\ pierald@gmail.com
}

\begin{abstract}
The aim of this text is to devise a flexible and thoughtful way to venture so responsibly in the plot of multiple dimensions involved in the architectural design and our contemporary reality immersed in a digital stage. We propose the integration of scans social and cultural process, from the recognition of the significant and symbolic choreography describing the bodies to inhabit the space in the architectural design process, through scenic bios architectural models.
\end{abstract}

Keywords: Cultural choreography; Variability; Complexity; Morphogenesis.

\section{Introducción}

Este trabajo presenta más que una información técnica una propuesta reflexiva, con la necesidad de asumir una posición particular en el proceso de diseño arquitectónico frente a todas las posibilidades que brindan las tecnologías digitales, tecnologías digitales que se multiplican día a día por las necesidades del mercado y se implantan en los procesos de implantación de las sociedades latinoamericanas.

El diseño arquitectónico contemporáneo, está inmerso en una cultura de variables unificadoras, cada vez más global, trasnacional, multicultural; envuelto de una manera especial por las posibilidades formales que lleva el sustrato de la tecnología digital y la innovación, variabilidad, complejidad, hibrides, multiformalidad. Pero ¿Cuál es el significado de esas formas, y en qué dirección apuntan hacia la arquitectura latinoamericana, venezolana, regional y local?

Esta doble tensión hace pensar desde la perspectiva cultural y tecnológica en universalismos o en un multiculturismos, pero la universalidad no tiene por qué contradecirse con la particularidad, desde esta mirada nos preguntarnos ¿Es la arquitectura un fenómeno universal en su totalidad? Sin dudas el cuerpo del ser humano, es universal. Reconocer que es a través de su corporeidad, que el ser humano habita la arquitectura, es fundamental para ampliar nuestros patrones creativos $y$ de investigación.

La arquitectura y la cultura conforman una visión esencial del mundo y condicionan la coreografía del habitar del ser humano. La integración entre arquitectura, cultura y paisaje pueden crear bios espacios que acentúen las complejas dimensiones sobre las nociones de identidad, historia y sentido de lugar, para afianzar ese necesario sentido de pertenencia por parte de los usuarios, así como establecer las particularidades que permitan revelar una mirada crítica sobre las características que conforman nuestra arquitectura, cultura y paisaje.

Los planteamientos expuestos en este trabajo bajo el enfoque del estudio de la comunicación no verbal y su interrelación con el espacio arquitectónico, forman parten de la experiencia del Seminario Espacio, Cuerpo y Comunicación asesorado por el Dr. Fuenmayor en el año 2012, dentro de los estudios doctorales en Arquitectura de la Universidad del Zulia, Maracaibo - Venezuela, y busca proponer modelos bios escénico arquitecturales, que exigen una exploración desde la ciencia de la vida, modelos que permitan dar el paso definitivo de la ruptura con una lógica lineal para dar paso a una lógica compleja multisensorial e inconsciente de interrelaciones, tal como lo plantea Fuenmayor (2006), y reformar la visión de la arquitectura contemporánea.

\section{De la Morfogénesis a la Corporeidad}

En este momento, la tecnología digital (TD) ha facilitado al profesional de la arquitectura visualizar, conceptualizar, representar, modelar, calcular, programar y concretar formas hipercomplejas, a través de un lenguaje de códigos multiformales, híbridos y heterogéneos. En otras palabras, la TD ha sido un puente hacia otras técnicas de observación, imaginación, visualización y conceptualización formal en el diseño arquitectónico.

La propuesta arquitectónica contemporánea en el escenario digital se inspira en la incorporación de los procesos biológicos, de la naturaleza a través de procesos de diseño morfogenéticos (diseño paramétrico, algoritmo genético), inspiración que pareciera ser una garantía de una visión renovada de comprensión y compromiso del ser humano hacia la naturaleza, hacia lo ecológico, y la utilización racional de los recursos del planeta. 
Como parte de un constructo cultural, el diseño morfogenético representa un cambio de pensamiento en el proceso de diseño arquitectónico. Podría decirse que un estado de conciencia de lo científico y lo tecnológico, que es capaz de contribuir con una arquitectura basada en procesos que ofrece la posibilidad de combinar campos un tanto dispares anteriormente en la profesión (Barroso, 2010).

Por otra parte, lo Biológico en la arquitectura es un tema interesante en todos los aspectos sobre todo porque en él, el arquitecto ha encontrado a lo largo de la historia una salida a la inhumanización (Barroso, 2010), pero toda esta atención a los patrones tecnológicos extranjeros, puede llegar a seducir al profesional de la arquitectura latinoamericana a restar atención a las condiciones locales y dejar a un lado los procesos de biogénesis, sociogénesis y psicogénesis particulares, no solo del arte sino de la cultura del ser humano.

De cualquier manera cada gran salto en la historia de la arquitectura traerá consigo una reinterpretación, por ello la teoría y crítica nos plantea una ruptura con la vanalidad arquitectónica y los estilismos sin sentido (Barroso, 2010), por una arquitectura que responda a las nociones de identidad, historia y sentido de lugar, para afianzar ese necesario sentido de pertenencia por parte de los usuarios, una arquitectura que de un salto importante a otro estado de conciencia, no solo científico y tecnológico sino cultural y humano, en otras palabras que responda a una ética en la complejidad del diseño.

Para Bustos (2012: 43) la definición de una ética en la complejidad del diseño arquitectónico con tecnología digital, se inicia, "en el sentido de pertinencia de dicho producto en relación holográmica con el sistema socio-ciber-cultura que la contenga, así como la flexibilidad de la respuesta arquitectónica en cuanto adaptabilidad a la necesidad del ser que lo habite".

Los planteamientos que presentamos no pretenden equiparar una propuesta definitiva, están en proceso de estudio, y así llegar a proponer su implementación en el ámbito académico en correspondencia a los cambios en los proceso de diseño arquitectónico a partir del uso de la tecnología digital, exploraciones de los proceso culturales y sociales, desde el reconocimiento de la coreografía significante y simbólica que describen los cuerpos al habitar el espacio, que a nuestro entender, se pueden hacer e integrar dentro del proceso de diseño arquitectónico, a través de modelos bios escénicos arquitecturales.

Los principios teóricos de la comunicación no verbal, han presidido nuestra investigación, guían en parte los planteamientos de este trabajo, así como los lineamientos del semiólogo Fuenmayor, en su propuesta de modelos bios escénicos que a continuación se describe.

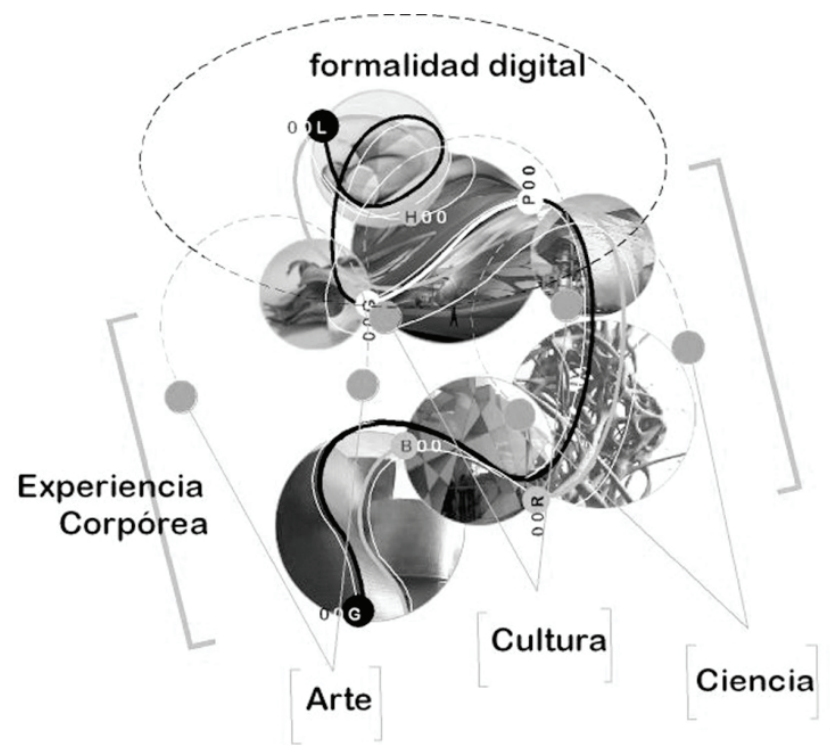

Figura 1: Abstracción del complejo entramado situacional implantado del escenario digital, Fuente Pirela (2013).

\section{Modelos Bios Escénicos}

Bios escénico es un modelo de investigación que propone Fuenmayor (2008:14), trata de relacionar las artes escénicas con un origen biológico, cultural y evolutivo de la especie humana cambiando el sentido dado hasta ahora al arte, al modelo humano y educativo y al concepto de cultura.

Fuenmayor (2010:5) formula reconocer la cultura como espacio escenográfico:

“Esa organización corporal inconsciente semejante a una partitura no escrita, una coreografía, que uno de los autores llama metafóricamente la danza de la vida (Edwar Hall) con ritmos entre mezclados comparables al tema de una sinfonía donde están implicados todos los aspectos del complejo comportamiento humano: kinésica (gestualidad), rítmico (temporalidad), proxémico (espacio y cuerpo) y la totalidad de la cultura (lenguaje, religión, filosofía, etc.)..."

La arquitectura, exige una exploración desde la ciencia de la vida. Es a través de su corporeidad que el ser humano habita la arquitectura, por ello reconocer el cuerpo como lo plantea Fuenmayor (2006) es fundamental para ampliar nuestros patrones creativos y de investigación, y proponer modelos bios escénicos arquitecturales, que estudien la intercompleja red de interacciones entre el arte, cultura y ciencia, lo relacional entre la diversidad cultural y la arquitectura, las bases biológicas de la comunicación, en el diseño del espacio arquitectónico como escenografía de la escena entre lo biológico y lo simbólico 


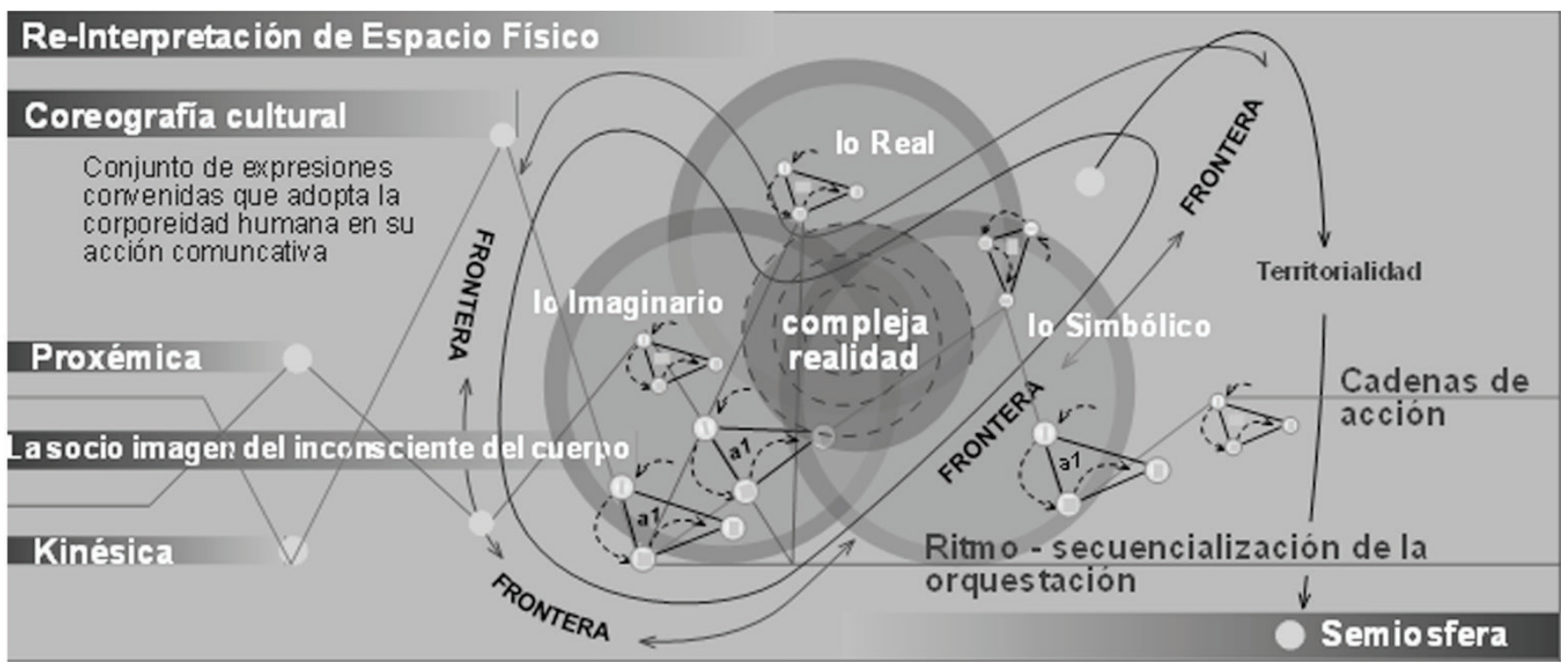

Figura 2: Esquema general, Lineamientos del modelo Bios escénicos arquitectural, Fuente Pirela (2013).

Por consiguiente, si nos situamos en una misma cultura, donde existe una coherencia en el esquema corporal cotidiano, que permita identificar los principios técnicos y escrituras corporales, y reconocer la socioimagen inconsciente del cuerpo, podríamos determinar a través de la captura digital la corporeidad de los usuarios y analizar la experiencia virtual visual de las plurales técnicas del cuerpo inmerso en una cultura, lo que amplia el sentido de humanización, en el abordaje digital del diseño arquitectónico.

A continuación se plantean los lineamientos del modelo bios escénicos arquitectural, estos describen las estrategias de reconocimiento coreográfico en la escena arquitectónica. (Ver figura 1).

\section{Lineamientos del Modelo Bios Escénicos Arquitectural}

"El cuerpo es el eje que nutre la interdisciplinaridad al poner en relación las ciencias que cubren los aspectos biológicos (perceptivos, neuronales, cerebrales) relevantes en las artes con las ciencias de la significación (Semiología, psicoanálisis, kinésica, proxémica, antropología"

(Fuenmayor, 2006:15)

La propuesta se basa en esa necesidad de desarrollar una sensibilidad especial sobre estos elementos intensamente cambiantes y aparentemente intangibles por la experiencia cotidiana, al identificar la socioimagen inconsciente del cuerpo, estímulo y la activación de la atención del usuario. Para que puedan ser sentidos no sólo por nuestros ojos sino también por nuestra psique y nuestro espíritu.

En primer lugar se recomienda profundizar sobre las redes complejas de las relaciones significantes que determina el espacio arquitectónico sobre el ser humano a través de su corporeidad cultural, es necesario una documentación básica sobre las teorías de la comunicación no verbal desarrolladas por los autores Edward Hall, Victor Fuenmayor, Yuri Lotman y Roland Barthes, en especial los conceptos de la dimensión oculta de la cultura, como corporeidad, kinésica, proxémica, semiosfera, frontera, entre otros.

Luego se aplica la teoría de la comunicación no verbal para el reconocimiento del espacio físico arquitectónico, se identifican las distintas semiosferas que se conforman en el espacio físico, a través de una observación reflexiva contemplativa sin llegar a interactuar con el usuario, ya buscamos penetrar el complejo laberinto de las multidimensiones ocultas e implicaciones que determina el espacio arquitectónico sobre el usuario a través de sus manifestaciones implícitas, inconscientes de su corporeidad, determinadas más por leyes artísticas o poéticas que por acciones conscientes y lingüísticas.

"El espacio de la semiosfera tiene un carácter abstracto". Lotman (1991:4) Significa que el concepto de espacio que emplea Lotman es en un sentido metafórico. Trata con una determinada esfera que posee los rasgos distintivos que se atribuye a un espacio cerrado en sí mismo. Sólo dentro de tal espacio resultan posibles la realización de los procesos comunicativos y la producción de una nueva información.

En este paso se describen los rasgos distintivos que caracterizan la semiosfera, ligado a su determinada homogeneidad e individualidad semiótica, su carácter delimitado, respecto del espacio extrasemiótico o alosemiótico que la rodea. Identificamos el tipo de frontera que las delimita. Puesto que el espacio de la semiosfera tiene un carácter abstracto, no debemos imaginarnos la frontera mediante los recursos de la imaginación concreta. Es una interpretación abstracta de la realidad concreta del movimiento corporal del usuario en el espacio arquitectónico. 
En este nivel estamos preparados para analizar las acciones comunicativas donde se encuentran representadas actitudes, conductas e interacciones de los usuarios, es decir, su corporeidad en la apertura y conciencia relacional con el escenario arquitectónico. Es decir, identificar las distintas particularidades conductuales del usuario en el espacio de estudio y así describir la coreografía en el llamado "orden simbólico".

La corporeidad así como el espacio material e inmaterial define el escenario donde las personas interactúan, determinando las distintas coreografías que conforman la vida social y promueven además un ambiente sicológico y socio-cultural que reafirma el sentido para la convivencia y el desarrollo de diversas actividades primarias, como características particular de los espacios arquitectónico.

La coreografía engloba el conjunto de expresiones convenidas socialmente que adopta la corporeidad humana en su acción comunicativa hacia el entorno, caracterizado por la coexistencia de respuestas de su realidad física local y de su memoria semántica.

El espacio como escenario para la coreografía de los usuarios trasciende a entorno físico, ya que su comportamiento tienen incidencia también por el entorno sicológico y socio-cultural, son textos que contienen mensajes indicativos de las coreografías que corresponde a un escenario particular.

Dentro de este contexto se efectúa la observación reflexiva contemplativa del comportamiento de los usuarios, para así describir las proxemicas, kinésicas y Ritmos- Secuencias del movimiento, que conforman la orquestación de la coreografía revelada.

En ese mismo sentido, la humanización por las técnicas del cuerpo no solo se trata de un proceso acumulativo de información sino organizativo y de selección que explica la individualidad y rasgos culturales. Esa coreografía determinante de comportamientos, semejante a una orquestación, correspondiente a la comunicación no verbal, partitura no escrita de los comportamientos sociales o la dimensión oculta de la cultura de los usuarios en estudio.

\section{Consideraciones Finales}

En esencia el oficio del arquitecto, es el saber interpretar, diseñar y construir el habitad del ser humano. Por ello, resulta necesario, reflexionar (examinar, rastrear e inspeccionar) sobre el modo como esta disciplina, esta practica constructiva y creadora, es concebida desde la tecnología digital, y más aun, reconocer y estudiar los efectos y las secuelas, que esas concepciones han traído consigo: lo que han hecho posible, e igualmente lo que han impedido.

Toda la arquitectura edificada es obligatoria para todos y es el marco en cual nos desarrollamos y vivimos. La arquitectura al igual que la escena, debe buscar un método en la interdisciplinaridad, en la transversabilidad del conocimiento, y buscar modelos bios escénicos arquitecturales, que estudien la intercompleja red de interacciones entre el arte, cultura y ciencia, lo relacional entre la diversidad cultural y la arquitectura, las bases biológicas de la comunicación, en el diseño digital del espacio arquitectónico como escenografía de la escena entre lo biológico y lo simbólico.

Re-valorar que la habitabilidad de la arquitectura es a través de la corporeidad de los usuarios y que el espació arquitectónico es un escenario coreográfico donde existe una coherencia en el esquema corporal cotidiano, e insertar dentro de los nuevos procesos de diseño bajo la visión de la multiplicidad los principios técnicos y escrituras corporales identificados en los modelos bios escénicos arquitecturales, amplia el sentido de humanización y las intenciones de la arquitectura.

Conocer la cultura a través del cuerpo permitirá al profesional de la arquitectura a: Identificar las necesidades específicas de quienes van a utilizar el espacio, el conocimiento de los usuarios mas allá de la formalidad de la arquitectura; Re-conocer los textos que integran la cultura del entramado situacional del emplazamiento de la obra arquitectónica y las practicas significantes de los futuros espacios; y una re-valorización de los principios humanos transculturales de la arquitectura.

\section{Referencias}

Barroso, N. (2010). Morfogénesis digital, Parametricismo y Arquitecturas Genéticas, Nuevas tendencias y formas de Proyectar Arquitectura. Trabajo de investigación Final de Becaría académica. Facultad de Arquitectura y Diseño. Universidad del Zulia. Maracaibo, Venezuela.

Bustos, G. (2012): Ética en la Complejidad del Diseño Arquitectónico con Tecnología Digital (TD): redes multidimensionales. XVI Congreso de la Sociedad Iberoamericana de Gráfica Digital. Universidad Federal de Ceará - Universidad de Fortaleza. SIGRADI Fortaleza. Brasil. 43-46.

Fuenmayor, V. (2010, julio/diciembre 8). Corporeidad, semiosis y memoria. Situarte, Revista Arbitrada de la Facultad Experimental de Arte de la Universidad del Zulia. Maracaibo, Venezuela. Año 5, 20-37.

Fuenmayor, V. (2006, julio/diciembre 1). El Bios escénico. Revista Arbitrada de la Facultad Experimental de Arte de la Universidad del Zulia. Maracaibo, Venezuela. Año 1, 13 - 22.

Lotman, Y. (1991 julio - diciembre 30). Acerca de la semiosfera, Criterios, II Época, 2 - 22. 